\title{
Enfoques de integración de información para sistemas \\ de monitoreo de salud estructural de puentes
}

\section{Approaches to Information Integration in Structural Health Monitoring Systems for Bridges}

César Garita ${ }^{1}$

Fecha de recepción: 28 de enero del 2015

Fecha de aprobación: 16 de junio del 2015

Garita, C. Enfoques de integración de información para sistemas de monitoreo de salud estructural de puentes. Tecnología en Marcha. Vol. 29, № 1, Enero-Marzo. Pág 96-107. 


\title{
Palabras clave
}

Integración de información; revisión; monitoreo de salud estructural; puentes.

\section{Resumen}

El diseño y desarrollo de sistemas que permitan recolectar, procesar, almacenar e integrar información asociada al monitoreo periódico de la condición de estructuras civiles como edificios y puentes, representa un gran reto desde el punto de vista computacional. En la práctica, podría ser necesario integrar un gran número de herramientas y componentes, con el fin de apoyar la toma ágil de decisiones estratégicas sobre el estado de salud de dichas estructuras por parte de los usuarios finales, incluyendo ingenieros y funcionarios de gobierno.

Muchos de estos componentes computacionales en general existen o han sido desarrollados previamente por diferentes organizaciones y exhiben un alto grado de heterogeneidad en cuanto a los modelos de datos y el software que utilizan. Este artículo presenta una revisión de enfoques de integración de información en áreas relacionadas con el monitoreo de la salud estructural, con énfasis en puentes. El estudio realizado busca recopilar experiencias que eviten "reinventar la rueda", así como identificar oportunidades de diferenciación, que sirvan de base para proponer enfoques novedosos de =integración de la información en este campo. Este trabajo se realizó como parte del proyecto de investigación e-Bridge 2.0, del Instituto Tecnológico de Costa Rica.

\section{Keywords}

Information integration; survey; structural health monitoring; bridges.

\begin{abstract}
The design and development of systems aimed at gathering, processing, storing and integrating information associated to periodic monitoring of the condition of civil structures such as buildings and bridges, represent a challenge from a computational point of view. In practice, there exist countless tools and components that may need to be integrated in order to support agile strategic decision making regarding structural health conditions. Many of these computational components may already exist or have been developed by different organizations and present a high level of heterogeneity in terms of data models and software configurations. This paper provides a study of information integration approaches in areas related to structural health monitoring with emphasis on bridge structures. The proposed study aims at putting together experiences that may avoid the "reinvention of the wheel" as well as the identification of differentiating aspects which may serve as a base for innovative approaches for information integration in this area. This work has been carried out as part of the e-Bridge 2.0 research project at the Costa Rica Institute of Technology.
\end{abstract}

\section{Introducción}

El Monitoreo de Salud Estructural (conocido como SHM, siglas en inglés de Structural Health Monitoring) puede describirse como el registro permanente, continuo o periódico de parámetros que reflejan la condición y el rendimiento de una estructura civil (Glisic e Inaudi, 2007). Por ejemplo, los parámetros pueden asociarse con valores de vibración medidos a través de un sensor asociado a una viga de un puente, durante una serie de pruebas de tránsito 
vehicular. La información obtenida a través del monitoreo se usa generalmente para planear y diseñar actividades de mantenimiento, aumentar la seguridad, verificar hipótesis y ampliar el conocimiento sobre la estructura en cuestión. El monitoreo contribuye a prevenir el impacto social, económico, ecológico y estético que pueda causar una falla en una estructura.

Con respecto a las herramientas computacionales y los sistemas de información que pueden utilizarse para apoyar el monitoreo y la subsecuente toma de decisiones en diversos ámbitos estratégicos, existe una amplia variedad de posibles soluciones y en la práctica las organizaciones que trabajan en este tema usan herramientas diferentes.

En el caso particular del monitoreo de la salud de los puentes, el sistema SHM, idealmente, debería apoyar procesos integrados para la toma de decisiones a través de funcionalidades tales como: interfases web amigables, servicios de Sistemas de Información Geográfica (SIG), unidades de monitoreo continuo, procesos de recolección y administración de datos, bases de datos históricas, sistemas de inteligencia de negocios, integración de sistemas existentes de información técnica de puentes, etc.

Por lo tanto, la adecuada integración de la gran cantidad de información generada por estos sistemas heterogéneos para que pueda ser procesada y utilizada de manera efectiva por ingenieros o tomadores de decisiones de las diferentes instituciones, representa un desafío desde el punto de vista computacional.

En este contexto, este artículo presenta una revisión de enfoques de integración de información en áreas relacionadas con monitoreo de la salud estructural, con énfasis en estructuras de puentes.

Este trabajo se desarrolló como parte del proyecto e-Bridge 2.0 - Sistema de información integrado para determinación del desempeño de estructuras de puentes, que reúne a profesionales de varias disciplinas, incluyendo ingeniería en construcción, computación, electrónica y producción industrial y forestal en el Instituto Tecnológico de Costa Rica (TEC) (Garita \& Ortiz, 2014). El objetivo principal de e-Bridge 2.0 es desarrollar un prototipo de sistema integrado de información para consultas estratégicas sobre el desempeño de los puentes, basado en datos obtenidos por medio de SIG, sensores de medición del desempeño, modelos de confiabilidad estructural e información técnica. Cada uno de estos sistemas utiliza modelos y herramientas de software diferentes que requieren su integración a nivel de datos, con el fin de apoyar consultas y manipulación integral de información.

El objetivo principal de este trabajo es hacer un estudio del estado del arte de áreas relacionadas con SHM de puentes desde el punto de vista específico de integración de información, con el propósito final de comprender los conceptos y funcionalidades generales asociados a componentes que deberán ser integrados en proyectos de monitoreo de salud de puentes tales como e-Bridge 2.0. Además, el estudio propuesto busca recopilar experiencias que eviten "reinventar la rueda" así como identificar oportunidades de diferenciación de nuevos enfoques de integración de información en SHM.

El análisis de los enfoques relacionados con la integración de información en SHM de puentes se ha dividido en las siguientes áreas de estudio:

a. Monitoreo de salud estructural de puentes: esta área representa el marco teórico del dominio de aplicación del sistema de integración.

b. Sistemas de monitoreo de puentes: en esta área se estudian las funcionalidades y enfoques generales de los sistemas de monitoreo de puentes existentes.

c. Administración de información en SHM: este punto incluye el estudio de modelos de información, bases de datos y sistemas administradores de datos en SHM de puentes. 
d. Integración con componentes específicos: esta categoría revisa enfoques relacionados con los componentes específicos de una arquitectura de referencia de los sistemas de SHM de puentes.

e. Integración de información en redes colaborativas: esta categoría se refiere a un estudio de modelos de redes colaborativas aplicables a la integración de información en SHM.

En las siguientes secciones se ofrece un resumen del análisis de trabajos relacionados con cada área.

\section{Monitoreo de salud estructural de puentes}

En esta sección se revisan los conceptos generales, las revisiones del estado del arte y metodologías de salud estructural de puentes, como base para comprender y analizar los requerimientos de integración de información en este tipo de sistemas.

\section{Conceptos generales}

Teniendo en cuenta la definición del concepto de SHM dada anteriormente en este artículo, entre los beneficios más comúnmente citados de SHM en general se pueden incluir (Bisby, 2004): mejor comprensión del comportamiento in situ de la estructura, detección temprana de daños, garantía de fortaleza y capacidad de servicio de la estructura, reducción del tiempo fuera de servicio, mejor mantenimiento y estrategias de asignación de recursos. En Wenzel (2009c) se presenta una visión general del monitoreo de la salud de puentes como un sistema integrado de apoyo a decisiones, basado en la web y con una interfaz amigable, que contiene diferentes elementos. En resumen, la arquitectura del sistema SHM está compuesta por sensores que generan datos de monitoreo, una base de datos que sirven para evaluar la condición de la estructura y generar alertas de eventos, así como diferentes sistemas de apoyo a la toma de decisiones.

\section{Estudios del estado del arte}

Existen varios estudios sobre el estado del arte en monitoreo de salud estructural de puentes. El reporte de Ahlborn et al. (2010) estudia el estado de la práctica de SHM y ofrece un resumen de las tecnologías existentes, incluyendo sensores y redes in situ, técnicas remotas y estudios de casos de puentes instrumentados.

En Enckell (2011) se presentan tecnologías establecidas y emergentes así como ejemplos relacionados con SHM y tecnologías de sensores para monitoreo de estructuras civiles, enfocándose en puentes. El libro de Wenzel (2009c) se concentra en prácticas actuales y metodologías de monitoreo dinámico de puentes.

\section{Metodologías}

Existen diversas metodologías para la determinación del nivel de riesgo estructural de los puentes. El esquema de clasificación BRIMOS (Wenzel, 2009a) se basa en cinco niveles, dependiendo del grado de profundidad de la investigación, incluyendo: calificación (rating), evaluación de condición, evaluación de rendimiento, evaluación detallada y predicción de vida útil. BRIMOS define una matriz de riesgo que relaciona el resultado de la evaluación del puente (muy bueno, bueno, razonable, moderado, bajo) con el impacto o consecuencia en la estructura (bajo, poco, moderado, serio, muy serio).

Según Dong y Song (2010), un sistema de SHM usualmente tendrá los siguientes componentes o pasos de una metodología demonitoreo: adquisición de datos de sensores, comunicación de 
datos, procesamiento y análisis de datos, almacenamiento de datos procesados, diagnóstico y pronóstico y acceso a datos. En el caso de e-Bridge (Fase I), la metodología propuesta de evaluación de puentes incluye principalmente los siguientes pasos: inventario (registro de datos técnicos), inspección visual, análisis y priorización, inspección detallada, pruebas de carga, monitoreo y análisis de confiabilidad.

\section{Sistemas de monitoreo de puentes}

El área de sistemas de monitoreo de puentes se divide en dos: revisiones generales de sistemas y sistemas específicos de SHM de puentes.

\section{Revisiones de sistemas}

En Gastineau, Johnson y Schultz (2009) se resumen los tipos de sistemas que están disponibles comercialmente para el monitoreo de la salud de los puentes. El reporte explica los criterios necesarios para determinar cuál sistema resultará más apropiado según las necesidades de los administradores de puentes. El trabajo reportado en Dong y Song (2010) tiene como objetivo sintetizar el conocimiento y las tecnologías actuales disponibles para monitoreo de infraestructuras civiles, y simplificar el proceso de seleccionar sistemas SHM para aplicarlos a estructuras de puentes.

\section{Sistemas específicos de SHM en puentes}

BRIMOS (Bridge Monitoring System) ofrece un método para la identificación de sistemas y detección de daño en puentes y otras estructuras civiles, basado en la respuesta dinámica a excitaciones del ambiente tales como viento, tráfico y actividades microsísmicas (Wenzel, 2009b). En resumen, la tecnología se basa en el monitoreo de las vibraciones en el ambiente y ofrece una amplia variedad de aplicaciones en SHM.

Por otro lado, el sistema SHM Live es un sitio web acoplado con una base de datos que administra y despliega datos de SHM monitoreados en tiempo real en cualquier lugar del mundo (Inaudi, 2012). Los servicios incluyen diseño, instalación, monitoreo y hardware por un monto mensual fijo. La base de datos puede recibir datos de muchos sistemas y sensores de medición. La información se incluye en la base central desde una unidad que se encarga a su vez de recolectar los datos directamente de todos los sistemas de adquisición de datos instalados.

\section{Administración de información en SHM}

El área de administración de información en SHM se divide en dos: modelos y bases de datos y sistemas administradores de datos.

\section{Modelos y Bases de datos}

En el campo particular de SHM y de estructuras civiles en general, hay poca estandarización en cuanto a modelos de datos, tecnologías, aplicaciones y herramientas. No obstante, existen prácticas que son comúnmente aceptadas por la comunidad de expertos. Algunos proyectos de investigación y desarrollo, tales como IRIS, han producido libros sobre seguridad industrial y ciclos de vida en ingeniería que proveen una base para la estandarización (Consortium, 2012). El proyecto SAMCO también dio lugar a que se escribieran libros que se han convertido en textos de referencia en este campo (Wenzel, 2009c). La herramienta GreenEyeData ofrece facilidades de despliegue y visualización básicas de datos de mediciones de SHM y cuenta con un estándar para especificar el formato de los datos de entrada (GreenEye, 2013). 
Sistemas administradores de datos

En Koo y Battista (2011) se presenta un sistema administrador de datos para SHM construido sobre MySQL para el almacenamiento, recuperación y compartición de conjuntos de datos extremadamente grandes adquiridos constantemente de sistemas SHM. El sistema también tiene una interfaz MATLAB y una interfaz web para facilitar el acceso a datos por parte de los investigadores usuarios.

En Tas (2010) se presenta una plataforma llamada Bridge Sensor Mart para la recolección, almacenamiento y análisis de datos de SHM en puentes. Entre los aspectos novedosos de este sistema está el uso de una base de datos híbrida (utiliza dos bases de datos diferentes) para aumentar la velocidad de acceso a los datos.

En Inaudi, Glisic y Vurpillot (2002) se describe la estructura de datos desarrollada en el contexto del proyecto SOFO. La estructura se basa en una BD relacional (Microsoft) e incluye un modelo básico de sensores y lecturas. Hay un módulo de adquisición de datos que permite navegar sobre la información en una BD centralizada. Luego, hay diferentes componentes que permiten hacer análisis sobre los datos almacenados.

\section{Enfoques de integración de componentes específicos}

Como se mencionó anteriormente, un sistema integrado de información para consultas estratégicas sobre desempeño de puentes se basa en datos obtenidos por medio de diferentes componentes incluyendo, p. ej., sistemas de información geográfica, medición de desempeño mediante sensores, modelos de confiabilidad estructural e información técnica de la estructura. Por tal motivo, en esta sección se hace una revisión de trabajos relacionados con cada uno de estos componentes.

\section{Sistema de Información Técnica}

Los datos técnicos que se recopilan sobre un puente dependen directamente del estándar o guía de evaluación de puentes que se aplique según los propósitos que definan. Por ejemplo, en FHWA (1995) se describe la guía utilizada por el Departamento de Transporte de Estados Unidos para la evaluación y codificación de datos de puentes. Incluye códigos de ítems que son parte integral de la base de datos utilizada para reportes y consultas sobre el estado técnico de los puentes.

A continuación, se describen algunos proyectos relacionados con información técnica de puentes:

- NBI. Como proyecto relacionado, el National Bridge Inventory (NBI) (FHWA, 2013) es un sitio web que se ofrece como un servicio público a todos los interesados en la condición y administración de puentes en Estados Unidos. El NBI posee una colección de más de 600,000 puentes en vías públicas en ese país.

- AASHTOWare Bridge. La asociación estadounidense AASHTO (American Association of State Highway and Transportation Office) ofrece el producto AASHTOWare Bridge, que consiste en un conjunto de herramientas para inventario, inspección, evaluación y diseño de puentes (AASHTO, 2013).

- SAEP-Conavi. En Costa Rica, el Consejo Nacional de Vialidad (Conavi) ha desarrollado un Sistema para la Administración de Estructuras y Puentes (SAEP) (Monge, 2011). La idea es que en el futuro cercano el SAEP contenga un inventario de los puentes nacionales con información técnica detallada que apoye funciones estratégicas de planificación y mantenimiento. 
- Estudio JICA. La Agencia Japonesa de Cooperación Internacional (JICA) realizó hace algunos años un estudio, a solicitud del Gobierno de Costa Rica, sobre el desarrollo de capacidad en planificación, rehabilitación, mantenimiento y administración de puentes, basado en 29 puentes de carreteras nacionales (JICA, 2007). Entre los objetivos está el establecimiento de herramientas de mantenimiento de puentes con manuales y lineamientos.

Confiabilidad y modelos de interpretación de datos

En esta sección, se analizan algunos modelos o metodologías para detectar comportamientos anómalos en estructuras a partir de datos de mediciones.

En Posenato (2006) se presentan dos métodos para hacer interpretación de datos "libre de modelo" (permite detectar daños sin usar modelos de comportamiento sino que usan solo datos de sensores). Los algoritmos memorizan características de series de tiempo generadas por sensores para detectar anomalías en el comportamiento de estructuras civiles.

En Phillips y Spencer (2012) se ofrece una revisión de literatura sobre simulación híbrida (pruebas experimentales en conjunto con simulación numérica). Los autores presentan un enfoque avanzado para la evaluación dinámica del rendimiento de sistemas estructurales utilizando simulación híbrida. En Bonigo (2013) se presenta un enfoque basado en la nueva área de "diagnosticabilidad" de sistemas, que podría tener aplicación en monitoreo y predicción de fallas en puentes.

El enfoque utilizado en la fase I de e-Bridge se utilizan índices de confiabilidad estructural basados en relaciones de condiciones reales (p. ej., deformaciones medidas durante pruebas de carga) versus teóricas de una estructura.

Desde el punto de vista de integración de la información, la conclusión principal es que los modelos interpretativos necesitan tener acceso a datos generados por sensores, queson procesados por sistemas estadísticos, los que a la postre producen indicadores específicos de riesgo o confiabilidad que a su vez deben almacenarse en el sistema integrado.

\section{Sistemas de información geográficos}

En esta sección se incluyen trabajos relacionados con geoportales, herramientas para el desarrollo de geoportales y SIG y algunos casos de aplicación de geoportales en escenarios estratégicos de toma de decisiones.

\section{Geoportales}

Existen muchas definiciones comunes y clasificaciones sobre el concepto de geoportal (Tait, 2005). Básicamente, se puede concluir que se trata de un portal que ofrece información o servicios almacenados en un SIG.

Tecnologías para desarrollo de geoportales

De igual forma, existen muchas iniciativas, herramientas y programas que posibilitan el desarrollo de portales con información geográfica (Association, 2012). Entre los más comunes se encuentran Open Geospatial Consortium (OGC), MapServer, GvSIG, PostGIS, GeoServer, MapFish, CartoWeb y MapBox.

Aplicación de geoportales en escenarios estratégicos

La aplicación de geoportales y SIG en escenarios que involucran decisiones estratégicas en distintas áreas socioeconómicas de interés nacional, tales como como manejo de desastres naturales, riesgo ambiental asociado al transporte, manejo de suelos, desarrollos urbanísticos, 
cambios en microcuencas, etc., debe tenerse en cuenta como referencia general en proyectos de monitoreo de la salud de los puentes.

El estudio presentado en López y Guevara (2012) busca detectar los cambios de cobertura del suelo en una microcuenca urbana en Colombia, ocurridos en un lapso de casi 20 años y utilizando fotografías aéreas digitales, para conocer la relación entre la presión humana sobre la cobertura del suelo y sus impactos en los hábitats urbanos vitales. El caso descrito en Pau Aragó (2012) se refiere a la aplicación de SIG en administración de riesgos de desastres asociados a alimentos y agricultura en Etiopía. En Moliner Santisteve (2012) se documenta un estudio en el que se utiliza un SIG con indicadores de huella de carbono y huella ecológica, para evaluar el impacto ambiental del transporte de residuos sólidos urbanos en España.

\section{Sensores de monitoreo}

Entre las variables que usualmente monitorean los sensores en SHM se encuentran: carga sobre la estructura, deformación, temperatura, aceleración y video. Quizás los sensores más comunes en SHM son los de fibra óptica, pues presentan ventajas específicas tales como: son no conductivos (presentan inmunidad a la interferencia electromagnética y frecuencias de radio), estabilidad, conveniencia y flexibilidad (Navarro-Henríquez, 2014). En Enckell (2011) también se indica que el mercado de tecnologías de sensores y de sistemas de adquisición de datos está cambiando aceleradamente y se señalan como tecnologías emergentes: emisión acústica, tecnologías de radar, fotogrametría, monitoreo de corrosión, sistemas de pesaje en línea, termografía infrarroja o imágenes térmicas y textiles técnicos inteligentes.

\section{Integración de información en redes colaborativas}

Esta sección revisa trabajos relacionados con conceptos y aplicaciones de redes colaborativas así como algunas herramientas disponibles actualmente para la administración de procesos de negocio (Business Process Management - BPM) y flujos de trabajo (workflows).

\section{Conceptos y aplicaciones de redes colaborativas}

Una Red Colaborativa (CN, por las siglas en inglés de Collaborative Network) es una alianza constituida por varias entidades (p. ej., organizaciones, sistemas y personas) que son autónomas o independientes en cuanto a administración y políticas, están geográficamente distribuidas y son heterogéneas en términos de su ambiente operativo y metas, pero que colaboran para poder alcanzar metas comunes y cuyas interacciones son apoyadas por redes de computadores (Camarinha-Matos y Afsarmanesh, 2005). En este contexto, las entidades colaboran estrechamente con el fin de, p. ej., ofrecer productos y servicios por parte de la red colaborativa que se llevan a cabo por medio de procesos desarrollados en varias organizaciones o por diversas personas. El paradigma de redes colaborativas ha sido aplicado con éxito en dominios tan variados como manufactura industrial, servicios en turismo, laboratorios virtuales y cuidado de adultos mayores.

El escenario del monitoreo de salud estructural de puentes puede verse como uno de redes colaborativas, pues involucra la interacción de entidades como municipalidades, instituciones de gobierno, universidades, centros de investigación, empresas privadas, tomadores de decisiones, ingenieros, etc., que son autónomas, heterogéneas y distribuidas, y necesitan colaborar de cerca con el fin de alcanzar una meta común: el monitoreo efectivo de la salud de los puentes bajo análisis. Para poder habilitar el paradigma de $\mathrm{CN}$, se han propuesto varios modelos de referencia que describen diversas dimensiones y componentes de este tipo de redes. Uno de los elementos comunes de cualquier tipo de $\mathrm{CN}$ es un administrador de procesos de negocio (BPM, siglas de Business Process Manager) que se encarga de coordinar los 
procesos que llevan a cabo las entidades de la red para la consecución de la meta común. En el caso de SHM de puentes, es posible definir procesos de negocios entre organizaciones o bien a lo interno de una organización, como el TEC, para coordinar la interoperación de los componentes del sistema SHM, tales como sistema de información técnica, geoportal, módulo de confiabilidad y sensores.

Este tipo de escenario ha sido modelado exitosamente mediante $\mathrm{CN}$ y en particular enfoques de flujos de trabajo. Entre los principales sistemas o herramientas disponibles actualmente para implementar procesos de negocio o flujos de trabajo que tienen potencial para servir de base para el enfoque de integración de información en SHM de puentes, se encuentran: Joget Workflow, Taverna, Bizagi, WebRatio, Bonita, ARIS (ver también Ami \& Sommer, 2007).

\section{Otras áreas relacionadas}

En general, existen muchas otras áreas de la computación que poseen un alto potencial para ser aplicadas en integración de información en SHM de puentes. A continuación se ofrece una breve descripción de algunas de ellas y sus relaciones con SHM:

- Grandes datos (big data). Este concepto se refiere al análisis avanzado de grandes volúmenes de datos de formato variable, que son alimentados al sistema a altas velocidades y requieren tiempos de respuesta cortos (Alexandrov, Brucke y Markl, 2013). Claramente, SHM presenta características que lo hacen un campo de aplicación de grandes datos y sus tecnologías.

- Computación en la nube. Armbrust y Konwinski (2009) se refieren a la computación en la nube como las aplicaciones ofrecidas como servicios a través de internet así como el hardware y los sistemas computacionales en los centros de datos que proveen esos servicios. Las ventajas de este enfoque incluyen que los usuarios pueden acceder al servicio en cualquier momento y lugar sin tener que preocuparse por la administración del software y hardware requerido. En el caso de SHM, el sistema de administración de datos podría alojarse en la nube, incluyendo las funcionalidades para visualización, análisis estadístico, panel de indicadores de control, etc.

- Minería de datos e inteligencia de negocios. En Wenzel, Furtner y Clifton (2013) se presentan algunos métodos novedosos para automatizar el proceso de evaluación de estructuras y, en particular, muestra el enfoque hacia la aplicación de instrumentos de minería de datos. Se presentan algunos indicadores clave de rendimiento de estructuras de puentes, incluyendo: integridad, evaluación de fatiga, localización de daño y curva del ciclo de vida. Los indicadores corresponden a un modelo de inteligencia de negocios para apoyar la toma de decisiones.

- Inteligencia artificial y aprendizaje máquina. Existen muchos procesos en SHM que presentan problemas que podrían resolverse utilizando técnicas de inteligencia artificial y aprendizaje máquina, por ejemplo: extracción de las variables o indicadores (p. ej., grado de daño), predicción de fallas, filtrar información, coordinación de sensores inteligentes y sistemas expertos. Para este tipo de problemas, es posible usar técnicas computacionales como: redes neuronales, algoritmos y programas genéticos, redes bayesianas y árboles de decisión, entre muchas otras. Ver enfoques en Hernandez-Garcia y Sanchez-Silva (2007) acerca de las máquinas de aprendizaje para la detección de daños estructurales.

- Ontologías para integración semántica. Básicamente, las ontologías representan un vocabulario común que diferentes sistemas pueden utilizar para interoperar y comunicarse. En Küng, Sonnleitner, Stumptner, Kosorus y Anderlik (2013) se presenta un enfoque de integración ontológica de sistemas heterogéneos de apoyo a la toma de decisiones y al intercambio de conocimiento entre las instituciones involucradas en SHM. 


\section{Conclusiones y recomendaciones}

A continuación se destacan las conclusiones y recomendaciones principales derivadas del estudio realizado sobre trabajos relacionados con integración de información en SHM, clasificadas según las áreas de análisis definidas:

- SHM de puentes. El área de SHM es un campo extenso, complejo y muy rico en cuanto a su potencial para el desarrollo de aplicaciones computacionales novedosas. Existen varios estudios del estado del arte en esta área, sin embargo, no están muy actualizados y no se enfocan específicamente en aspectos de integración de datos. A pesar de que hay muchos sistemas y tecnologías para SHM, persisten retos importantes con respecto al manejo de grandes cantidades de información en tiempo real, así como en análisis y predicción de las condiciones de los puentes.

- Sistemas de monitoreo de puentes. Del estudio realizado, los proyectos de BRIMOS y SHMLive están entre los sistemas más completos e integrales para el monitoreo de puentes. Cabe mencionar que estos son proyectos comerciales y no son sistemas de código abierto.

- Administración de información en SHM. El SHM de puentes es un dominio que está muy poco estandarizado en cuanto a modelos y enfoques de administración de datos. En la mayoría de los enfoques revisados se utiliza una base de datos relacional centralizada para recopilar la información de las unidades que a su vez obtienen los datos de los sensores.

- Integración de componentes específicos. Los componentes específicos que deben integrarse para un sistema de monitoreo de puentes presentan enfoques y tecnologías muy heterogéneos desde el punto de vista computacional. La integración de información de estos componentes debe realizarse mediante un mecanismo lo más general posible, tomando en cuenta los altos niveles de heterogeneidad, distribución física y autonomía en el manejo de información que presentan estos sistemas. Se recomienda que el enfoque de integración esté basado en servicios web, para efectos de interoperabilidad, reutilización y escalabilidad del sistema.

- Integración de información en redes colaborativas. El paradigma de redes colaborativas es aplicable al área de SHM de puentes. Con base en el presente estudio, se puede concluir que ninguno de los enfoques y proyectos revisados aplica la disciplina de redes colaborativas y, en particular, ninguno aplica la administración de procesos de negocios con el fin de integrar información entre entidades o componentes. Se recomienda analizar la posibilidad concreta de utilizar modelos y herramientas de flujos de trabajo para este propósito.

- Otras conclusiones y recomendaciones. Existen muchas tecnologías computacionales relacionadas con administración de información en SHM que podrían aplicarse, incluyendo: big data, computación en la nube, minería de datos, inteligencia de negocios, etc. Sin embargo, se recomienda que estas tecnologías potenciales sean evaluadas en función del alcance, recursos y calendario en su debido contexto, antes de ser consideradas para su implementación.

Por último, el material sintetizado y analizado en este documento representa una base sólida para la toma de decisiones en proyectos de uso o desarrollo de sistemas de información en SHM de puentes. 


\section{Bibliografía}

AASHTO. (2013). AASHTOWare Bridge. Obtenido de http://www.aashtoware.org/Bridge/Pages/default.aspx

Ahlborn, T.M., Shuchman, R., Sutter, L.L., Brooks, C.N., Harris, D.K., Burns, J.W. \& Oats, R.C. (2010). The Stateof-the-Practice of Modern Structural Health Monitoring for Bridges: A Comprehensive Review. Michigan Technological University.

Alexandrov, A., Brucke, C. \& Markl, V. (2013). Issues in big data testing and benchmarking. Paper presented at the Proceedings of the Sixth International Workshop on Testing Database Systems - DBTest '13, New York.

Ami, T. \& Sommer, R. (2007). Comparison and evaluation of business process modelling and management tools. International Journal of Services and Standards, 3(2), 249-261.

Aragó, P., Kasie, T., Grandío, A., Sergio Clark, S. \& Huerta, J. (2012). GVSIG education for Disaster Risk Management within an international cooperation project. Paper presented at the 8as Jornadas Internacionales gvSIG, Valencia, España.

Armbrust, M., Griffith, R., Joseph, A.D., Katz, R., Konwinski, A., Patterson, D.A., Rabkin, A., Stoica, I. \& Zaharia, M. (2009). Above the Clouds: A Berkeley View of Cloud Computing (E. E. a. C. Sciences, Trans.). University of Berkeley.

Association, U.I.R.M. (2012). Geographic Information Systems: Concepts, Methodologies, Tools, and Applications.

Bisby, L.A. (2004). ISIS Educational Module 5: An Introduction to Structural Health Monitoring. ISIS Canada.

Bonigo, L.B. (2013). Diagnosability Behaviour over Faulty Concurrent Systems. Paper presented at the XXXIX Conferencia Latinoamericana en Informática - CLEI 2013, Naiguatá, Venezuela.

Camarinha-Matos, L.M. \& Afsarmanesh, H. (2005). Collaborative networks: a new scientific discipline. Journal of Intelligent Manufacturing, 16(439-452).

Consortium, I. (2012). Industrial Safety and Life Cycle Engineering - Technologies / Standards / Applications.

Inaudi, D., Glisic, B. \& Vurpillot, S. (2002). Database structures for the management of monitoring data. Paper presented at the Structural Health Monitoring Workshop, Winnipeg, Canada.

Dong, Y. \& Song, R. (2010). Bridges Structural Health Monitoring and Deterioration Detection. Synthesis of Knowledge and Technology. (D. o. C. E. Engineering, Trans.). University of Alaska Fairbanks.

Enckell, M. (2011). Lessons Learned in Structural Health Monitoring of Bridges Using Advanced Sensor Technology. Doctorate Ph.D. Thesis, Royal Institute of Technology, Sweden.

FHWA. (1995). Recording and Coding Guide for the Structure Inventory and Appraisal of the Nation's Bridges. Federal Highway Administration.

FHWA. (2013). The National Bridge Inventory. Obtenido de http://nationalbridges.com/nbi

Garita, C. \& Ortiz, G. (2014). Towards a Workflow Management Approach for Health Monitoring of Bridges. Paper presented at the 15th IFIP International Working Conference on Virtual Enterprises PRO-VE, Amsterdam, The Netherlands.

Gastineau, A., Johnson, T. \& Schultz, A. (2009). Bridge Health Monitoring and Inspections. A Survey of Methods. University of Minnesota.

Glisic, B. \& Inaudi, D. (2007). Fibre Optic Methods for Structural Health Monitorin. 1 ed. John Wiley \& Sons.

GreenEye. (2013). Green Eye Data. Obtenido de http://www.green-eye-data.com/default_en.htm

Hernandez-Garcia, M. \& Sanchez-Silva, M. (2007). Learning Machines for Structural Damage Detection. En N. Lagaros y Y. Tsompanakis (Eds.), Intelligent Computational Paradigms in Earthquake Engineering. IGI Global.

Inaudi, D. (2012). SHMLive Web-based Data Management Software.

JICA. (2007). El estudio sobre el desarrollo de capacidad en la planificación de rehabilitación, mantenimiento y administración de puentes basado en 29 puentes de la red de carreteras nacionales en Costa Rica. Resumen de Informe Final.

Koo, K.Y. \& Battista, N.D. (2011). SHM Data Management System Using MySQL Database with MATLAB and Web Interfaces. Paper presented at the 5th International Conference on Structural Health Monitoring of Intelligent Infrastructure (SHMII-5), Cancún, México. 
Küng, J., Sonnleitner, E., Stumptner, R., Kosorus, A.H. \& Anderlik, S. (2013). Utilizing Ontologies to Integrate Heterogeneous Decision Support Systems. En H. Wenzel (Ed.), Industrial Safety and Life Cycle Engineering. Technologies / Standards / Applications.

López, J. \& Guevara, C. (2012). Análisis del cambio de tipo de cobertura en la microcuenca urbana Hato de la Virgen ubicada en el municipio de Ibague. Paper presented at the 8as Jornadas Internacionales gvSIG, Valencia, España.

Moliner Santisteve, R.V.N., Garraín Cordero, D., Gasch García, C. \& Muñoz Suárez, I. (2012). Aplicación de GVSIG en un Estudio de Evaluación de Impacto Ambiental del Transporte de Residuos. Documento presentado en las 8as Jornadas Internacionales gvSIG, Valencia, España.

Monge, A. (2011). Manual de Instalación de Versión Educativa del Sistema de Administración de Estructuras y Puentes (SAEP). San José: Conavi.

Navarro-Henríquez, F. (2014). Sensores de fibra óptica FBG para el monitoreo de la salud estructural de los puentes. Tecnología en Marcha, 27(4).

Phillips, B.M. \& Spencer, B.F.,Jr. (2012). Model-Based Framework for Real-Time Dynamic Structural Performance Evaluation. Newmark Structural Engineering Laboratory Report Series 031.

Posenato, D., Lanata, F., Inaudi, D. \& Smith, I.F.C. (2006). Model Free Interpretation of Monitoring Data. Intelligent Computing in Engineering and Architecture, 4200.

Tait, M. (2005). Implementing geoportals: applications of distributed GIS. Computers, Environment and Urban Systems, 29(1).

Tas, N.C., Dejori, M. \& Neubauer, C. (2010). Bridge Sensor Mart: A Flexible and Scalable Data Storage and Analysis Framework for Structural Health Monitoring. Paper presented at the 5th International Conference on Bridge Maintenance, Safety and Management, Philadelphia, USA.

Wenzel, H. (2009a). From Structural Health Monitoring to Risk based Infrastructure Management. Paper presented at the 4th International Conference on Structural Health Monitoring on Intelligent Infrastructure (SHMII-4) 2009, Zurich, Switzerland.

Wenzel, H. (2009b). From Structural Health Monitoring to Risk based Infrastructure Management. Paper presented at the 4th International Conference on Structural Health Monitoring on Intelligent Infrastructure - SHMII-4 2009, Zurich, Switzerland.

Wenzel, H. (2009c). Health Monitoring of Bridges (1st ed.): Wiley.

Wenzel, H., Furtner, P. \& Clifton, R. (2013). The Role of Structural Health Monitoring in the Life-Cycle-Management of Bridges. Paper presented at the 8th International Cable Supported Bridge Operators Conference - ICSBOC 2013, Edinburgh, Scotland. 